\title{
Physiological Characteristics and Vitamin Requirements of Lactobacilli Isolated from Milk and Cheese
}

\author{
BY J. G. FRANKLIN AND M. ELISABETH SHARPE \\ National Institute for Research in Dairying, \\ University of Reading
}

(Received 23 August 1963)

SUMMARY

The vitamin requirements and physiological characteristics of 179 strains of lactobacilli isolated from milk and cheese have been studied and their relationship to classification discussed. The vitamin requirements showed good correlation with the results of the physiological and biochemical tests with strains of the subgenus Streptobacterium but the correlation was poor among the beta bacteria. It was not possible to differentiate the physiologically similar Lactobacillus brevis and $L$. buchneri species by their vitamin requirements. The nutritional data indicated that the species L. plantarum probably consists of more than one variety, and confirmed the reliability of a thiamine requirement as a means of differentiating heterofermentative and homofermentative lactobacilli. The nutritional data also proved useful with some strains by verifying their unclassifiable status or by making possible their classification when they could not be identified with certainty on the basis of biochemical tests alone. The nitratase activities of strains of $L$. casei, L. plantarum, L. brevis, L. buchneri and pediococci were also studied. Four of the L. plantarum and 2 of the $L$. casei strains were able to reduce nitrate but none of the other species tested showed this characteristic.

\section{INTRODUCTION}

In a recent study of the bacterial flora of cheese milk and Cheddar cheese (Franklin \& Sharpe, 1963), 1755 strains of lactobacilli were isolated and classified by using a variety of biochemical and physiological tests. Whilst most of these strains were satisfactorily assigned to appropriate species, a few strains could not be identified because their biochemical characteristics were intermediate between species. Rogosa and co-workers $(1947,1953,1961)$ found that the biochemical and cultural characteristics of members of the genus Lactobacillus correlated well with their vitamin requirements. Accordingly, the vitamin requirements of representative strains of these milk and cheese isolates were investigated to determine whether a similar correlation occurred with these organisms and also to see whether knowledge of their vitamin requirements assisted in the identification of the unclassified strains.

Costilow \& Humphreys (1955) observed that some strains of Lactobacillus plantarum reduced nitrates under certain conditions and this was later confirmed by Rogosa (1961). Because of the ready availability, in this investigation, of a large number of strains of lactobacilli and pediococci from different sources, the oppor- 
tunity was taken of examining these organisms for nitratase activity to determine the extent to which these observations were confirmed. The strains tested, however, were not always the same as those used in the nutritional study.

\section{METHODS}

Lactobacilli isolated from milk and cheese as described by Franklin \& Sharpe (1963) were classified according to the schemes of Rogosa \& Sharpe (1959) and Perry \& Sharpe (1960), by using the following tests: microscopic appearance; catalase production; growth at $15^{\circ}$ and $45^{\circ}$; production of gas from glucose; production of ammonia from arginine; splitting of aesculin; growth in $0.1 \%$ Teepol (Shell Chemical Co. Ltd., Marlborough House, 15-17 Great Marlborough St, London W. 1.) and fermentation of sugars. Cultures were grown in MRS broth (de Man, Rogosa \& Sharpe, 1960) and washed suspensions were used for inoculation of the test media.

In addition to the above tests, some strains were also tested for the reduction of nitrate using a medium of low carbohydrate content maintained at a relatively high $\mathrm{pH}$ within the nitratase activity range (Rogosa, 1961). The composition of this medium was as follows $(\%, w / v)$ : Oxoid peptone, 1.0; Oxoid Lab-Lemco, 1.0; Oxoid yeast extract, 0.5 ; glucose, $0 \cdot 2$; Tween 80 (Polyoxyethylene sorbitan oleate, Honeywill and Stein Ltd., Devonshire House, Mayfair Place, Piccadilly, London W. 1) $0 \cdot 1 ; \mathrm{K}_{2} \mathrm{HPO}_{4}, 0 \cdot 2 ; \mathrm{CH}_{3} \mathrm{COO} \mathrm{Na} .3 \mathrm{H}_{2} \mathrm{O}, 1 \cdot 0$; tri-ammonium citrate, $0 \cdot 2 ; \mathrm{MgSO}_{4} .7 \mathrm{H}_{2} \mathrm{O}$, $0 \cdot 02 ; \mathrm{MnSO}_{4} \cdot 4 \mathrm{H}_{2} \mathrm{O}, 0 \cdot 005 ; \mathrm{KNO}_{3}, 0 \cdot 1$. The $\mathrm{pH}$ value was adjusted to $7 \cdot 5-7 \cdot 6$ before sterilization. The method of testing for nitrate reduction was that used by Rogosa (1961). The vitamin requirements of the lactobacilli were determined by using the medium and methods described by Rogosa, Franklin \& Perry (1961).

The taxonomy of the lactobacilli and many of the tests used were discussed recently by Rogosa \& Sharpe (1959) and by Sharpe (1962).

\section{RESULTS AND DISCUSSION}

The milk and cheese lactobacilli isolates which could be satisfactorily classified on the basis of physiological tests belonged to the four species L. casei, L. plantarum, L. brevis and L. buchneri. As it was not practicable to examine the vitamin requirements of all the $\mathbf{1 7 5 5}$ strains, representative typical strains were studied from each of these species, together with those strains which could not be classified biochemically into named species or which, although resembling a particular species sufficiently to warrant inclusion in that group, nevertheless differed from the typical reactions of that species in one or more important characteristics. For reasons of simplicity in this publication, the description 'typical' is applied to named strains showing the differentiating physiological characteristics or nutritional requirements of species of lactobacilli as described by Rogosa \& Sharpe (1959) and Rogosa et al. (1961).

Lactobacillus casei. The vitamin requirements were determined for 74 strains which were classified physiologically as $L$. casei or which resembled this species more than any other. Twenty-two per cent of these, giving the typical reactions of this species listed in Table 1 , were able to grow at $45^{\circ}$, whilst $14 \%$ of those giving the typical reactions did not grow at this temperature. Thirty-four per cent differed 


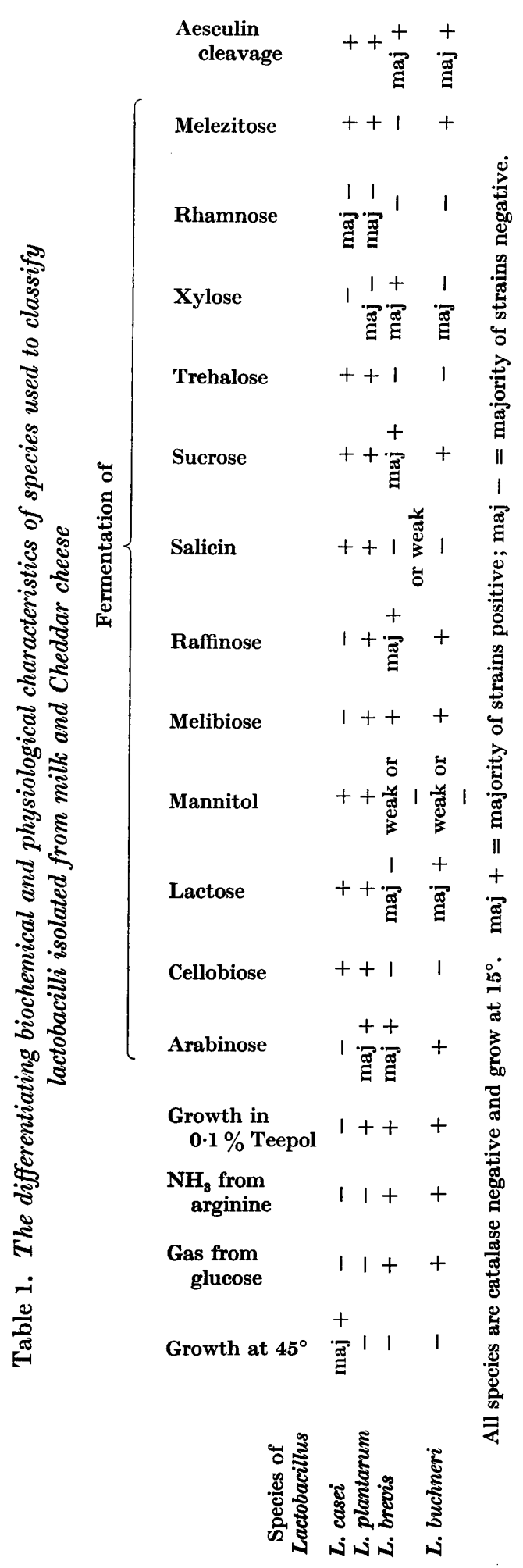


from the typical in one character, $26 \%$ in two characters, $3 \%$ in three characters and $1 \%$ in four characters. The extent to which the different tests gave atypical results with the 74 strains was as follows : sucrose negative, $33 \%$; trehalose negative, $23 \%$; cellobiose negative, $10 \%$; salicin negative $8 \%$; mannitol negative, $7 \%$; Teepol positive, $5 \%$; aesculin negative, $3 \%$; lactose negative, $3 \%$; melezitose negative, $3 \%$; rhamnose positive, $1 \%$; arabinose positive, $1 \%$.

The vitamin requirements for the 74 strains of Lactobacillus casei tested are given in Table 2. Most of the strains (91\%) had the typical vitamin requirements for the species, and no strain required thiamine. Only one strain had no requirement for riboflavin and this strain differed from the typical physiological and biochemical characteristics of the species only in its failure to ferment sucrose. The remaining strains having atypical vitamin requirements had no other atypical characteristics in common.

Table 2. The vitamin requirements of 121 strains of the sub-genus Streptobacterium Orla-Jensen

Nicotinic acid and pantothenic acid were required by all strains.

\begin{tabular}{|c|c|c|c|c|c|}
\hline Species & $\begin{array}{l}\text { No. of } \\
\text { strains }\end{array}$ & Thiamine & Riboflavin & Pyridoxal & Folic acid \\
\hline \multirow{7}{*}{$\begin{array}{l}\text { L. casei } \\
\quad \text { (74 strains) }\end{array}$} & 62 & - & + & + & $+*$ \\
\hline & $\mathbf{5}$ & - & + & $\mathbf{S}$ & $+*$ \\
\hline & $\mathbf{3}$ & - & + & $\mathbf{S}$ & - \\
\hline & $\mathbf{1}$ & - & + & + & - \\
\hline & 1 & - & + & - & + \\
\hline & 1 & - & - & + & $\mathbf{S}$ \\
\hline & 1 & - & + & - & - \\
\hline \multirow{4}{*}{$\begin{array}{c}\text { L. plantarum } \\
\text { (47 strains) }\end{array}$} & $\mathbf{3 0}$ & - & + & - & $-\dagger$ \\
\hline & 14 & - & - & - & -+ \\
\hline & 2 & - & - & $\mathbf{S}$ & - \\
\hline & 1 & - & + & $\mathbf{S}$ & - \\
\hline
\end{tabular}

Lactobacillus plantarum. The vitamin requirements were determined for 47 strains classified physiologically as, or closely related to, L. plantarum. Twenty-four per cent of these gave the typical physiological reactions listed in Table 1, 24\% differed from the typical physiological pattern by one character, $9 \%$ by two characters, $4 \%$ by three characters and $39 \%$ by four characters. The proportion of the 47 strains differing in particular characters from the typical were as follows: Teepol negative, $63 \%$; trehalose negative, $54 \%$; sucrose negative, $52 \%$; raffinose negative, $41 \%$; melibiose negative, $2 \%$ ( 1 strain). Unlike the $L$. casei group, no strains of $L$. plantarum were able to grow at $45^{\circ}$.

The vitamin requirements of the 47 strains of Lactobacillus plantarum tested are given in Table 2 . Nearly all the strains $(94 \%)$ had the typical vitamin requirements for the species although it is evident that there are two varieties of $L$. plantarum, one requiring riboflavin and one having no requirement for this vitamin. This confirms the findings of Rogosa et al. (1953). Three strains only differed from the typical patterns in showing a stimulatory action of pyridoxal. 
The strains which had no riboflavin requirement had the typical physiological characteristics of this species or differed only in their failure to grow in $0 \cdot 1 \%$ Teepol, whereas with the exception of two typical strains and one strain which did not grow in $0.1 \%$ Teepol, the remaining 27 strains which exhibited a requirement for riboflavin also differed from the typical physiological pattern in their failure to ferment one or more of sucrose, trehalose, raffinose and melibiose.

Table 3. The vitamin requirements of 46 strains of the subgenus Betabacterium Orla-Jensen

Nicotinic acid and pantothenic acid were required by all strains.

$\begin{array}{cccccc}\text { Species } & \begin{array}{c}\text { No. of } \\ \text { strains }\end{array} & \text { Thiamine } & \text { Riboflavin } & \text { Pyridoxal } & \text { Folic acid } \\ \text { L. brevis } & \mathbf{9} & + & + & - & + \\ \text { (27 strains) } & \mathbf{6} & + & \mathbf{S} & + & + \\ & 4 & + & + & + & + \\ & \mathbf{3} & + & - & - & +{ }^{*} \\ & \mathbf{3} & + & \mathbf{S} & + & + \\ \text { L. buchneri } & \mathbf{2} & + & + & - & + \\ \text { (19 strains) } & \mathbf{6} & + & - & - & -\dagger \\ & \mathbf{6} & + & - & \mathbf{S} & + \\ & & + & + & \mathbf{S} & +\end{array}$

* Typical vitamin requirement pattern for L. brevis strains (Rogosa et al. 1961).

† Typical vitamin requirement pattern for $L$. buchneri strains (Rogosa et al. 1961).

$+=$ requirement; - = no requirement; $S=$ stimulatory.

Lactobacillus brevis. Vitamin patterns were determined for 27 strains classified as L. brevis. Eight strains gave the typical physiological reactions for this species as listed in Table 1 and 8 strains differed by only one character (Teepol negative, 5 strains; melibiose negative, 6 strains; $\mathrm{NH}_{3}$ from arginine negative, 1 strain).

The vitamin requirements for the 27 strains of Lactobacillus brevis are given in Table 3 which shows that only three strains gave the typical vitamin requirement pattern for this species. All the strains possessed the typical thiamine requirement of the heterofermentative lactobacilli and all required folic acid, but this latter property was also found in other betabacteria. Contrary to the previous findings, riboflavin was essential for growth of, or was stimulatory to, 24, strains, and pyridoxal was essential for the growth of 13 strains. The atypical vitamin-requirement patterns obtained in this group were different combinations of the various vitamin requirements exhibited by so-called typical strains of $L$. brevis, $L$. buchneri and $L$. viridescens. All these strains had the physiological characteristics of $L$. brevis, however. None fermented melezitose and all differed in some respect from typical L. viridescens strains.

Lactobacillus buchneri. Vitamin requirements were determined for 19 strains classified physiologically as L. buchneri. Four strains gave the typical physiological characteristics for this species as shown in Table 1, 13 strains differed in one character from the typical and 2 strains in two characters. The proportions of the 19 strains differing in particular characters from the typical were as follows: Teepol negative, 13 strains; raffinose negative, 1 strain; sucrose negative, 1 strain; melezitose negative, 1 strain; salicin positive, 1 strain. 


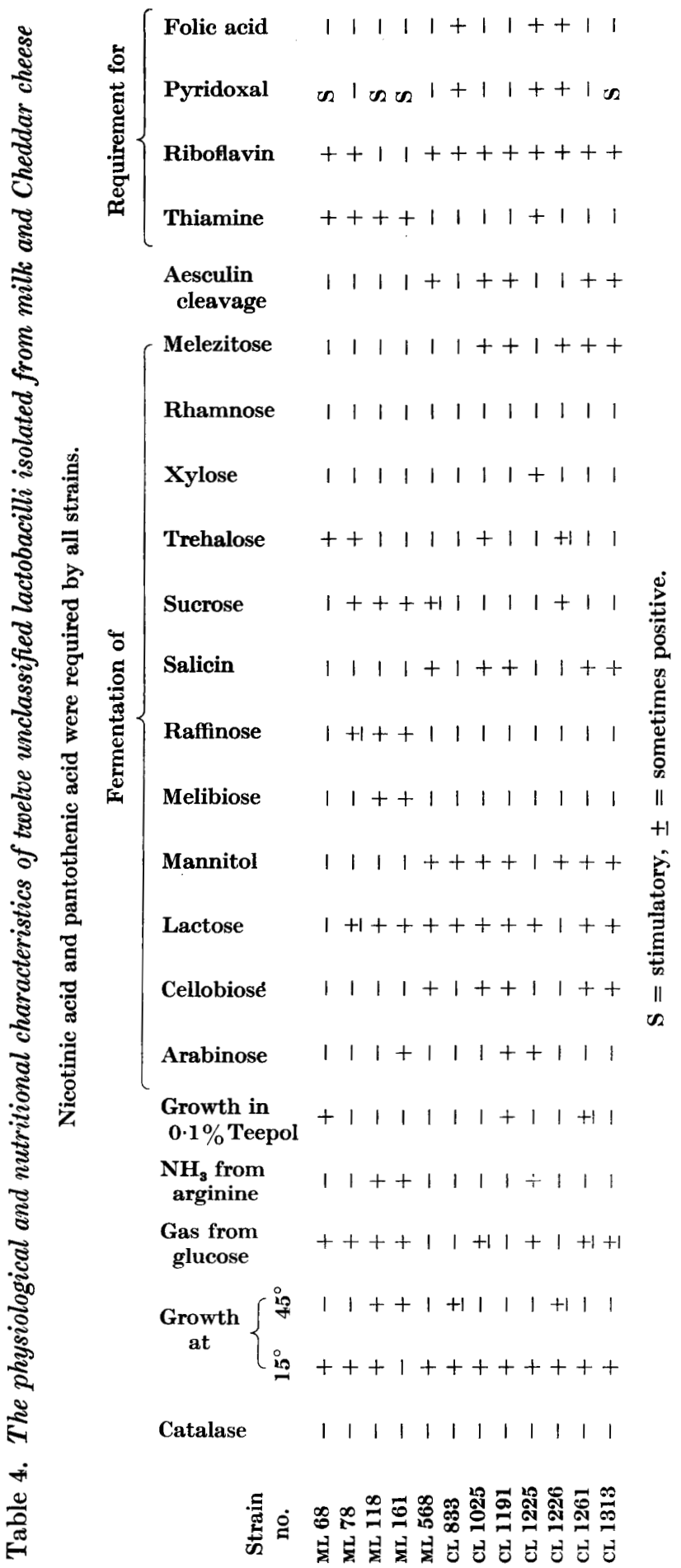


The vitamin requirements for the 19 strains of Lactobacillus buchneri are given in Table 3. All the strains required thiamine but only 7 strains exhibited the typical vitamin requirement pattern for this species. Pyridoxal was stimulatory and folic acid essential for the growth of the remaining 12 strains which could be equally divided into those which required riboflavin and those which did not. Some strains of $L$. buchneri have been observed to show a requirement for riboflavin and this is confirmed here, although in the present work this character was always accompanied by a requirement for folic acid. The atypical vitamin requirement patterns obtained in the group again appear to be different combinations of the so-called typical patterns for $L$. brevis, $L$. buchneri and $L$. viridescens. However, all the atypical strains produced ammonia from arginine and fermented melezitose, arabinose and melibiose, and could not, therefore, be classified either as $L$. brevis or L. viridescens species.

\section{Unclassified Lactobacillus strains}

Twelve strains were unclassified physiologically and their physiological and nutritional characteristics are given in Table 4. Some of the implications of these results in relation to their classification are discussed below.

Strain ML 68. Production of gas from glucose places this strain in the subgenus Betabacterium. Failure to produce ammonia from arginine, its inability to grow at $45^{\circ}$ and its relative non-fermentative nature suggest that it might be classified as $L$. viridescens and, except that folic acid was not stimulatory, this is supported by the nutritional data.

Strain ${ }_{M L}$ 78. This strain is also a member of the betabacteria but differs from ML 68 in its failure to grow in $0.1 \%$ Teepol, its fermentation of lactose and raffinose and the lack of growth stimulation by pyridoxal. This strain does not, therefore, fit into any of the recognized species in this subgenus.

Strain $M L$ 118. The physiological characters of this strain suggest that though capable of growth at $15^{\circ}$ it belongs to the species $L$. fermenti. The vitamin requirements supported this classification although pyridoxal was stimulatory contrary to the usual pattern for this group.

Strain ML 161 gave identical results to ML 118 except for its failure to grow at $15^{\circ}$, and would, therefore, also appear to belong to the species $L$. fermenti.

Strain $M L$ 568. The physiological results, with the exception of the failure to ferment trehalose, indicate that this strain is clearly a member of the species $L$. casei. This is not supported by the nutritional results, however, which were typical of L. plantarum.

Strain $C L$ 833. This fermented few carbohydrates but the typical vitamin requirements of $L$. casei exhibited by this organism suggest that CL 833 should be assigned to this species.

Strain oL 1025. With the exception of the production of a small amount of gas from glucose and the failure to ferment sucrose, this strain had the typical physiological reactions of $L$. casei; its vitamin requirements, however, were those of L. plantarum. The absence of a thiamine requirement excluded it from the heterofermentative lactobacilli and the physiological characteristics, with the exception of gas production, were unlike those of any named Betabacterium species. 
Strain $C L 1261$. This was very similar to CL 1025, having physiological characters closely related to $L$. casei, the vitamin requirements of $L$. plantarum, yet producing small quantities of gas from glucose.

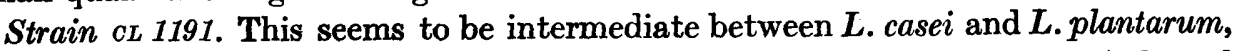
being unable to ferment melibiose and raffinose but able to grow in $0.1 \%$ Teepol and fermenting arabinose. The vitamin requirements, however, were typical of L. plantarum.

Strain CL 1225. Although clearly a member of the subgenus Betabacterium, this organism cannot be placed into any of the named species in this group; this was confirmed by the nutritional data.

Strain oL 1226. This physiologically inactive strain showed the typical vitamin requirements of $L$. case $i$ suggesting that it is most closely related to this species.

Strain cL 131. This strain resembles $L$. casei physiologically, although producing small volumes of gas from glucose, but this classification is not entirely supported by the nutritional pattern which was intermediate between those of $L$. casei and L. plantarum.

Table 5. Nitrate reduction by lactobacilli and pediococci isolated from milk and cheese

$\begin{array}{lcc}\text { Species } & \begin{array}{c}\text { No. strains } \\ \text { tested }\end{array} & \begin{array}{c}\text { No. strains } \\ \text { positive }\end{array} \\ \text { L. casei } & 102 & 2 \\ \text { L. plantarum } & 12 & 4 \\ \text { L. brevis } & 18 & 0 \\ \text { L. buchneri } & 8 & 0 \\ \text { Pediococci } & 9 & 0\end{array}$

The results of this investigation confirm the consistency that exists between the physiological characters of strains of Lactobacillus casei and $L$. plantarum and their vitamin requirements. The nutritional patterns obtained with $L$. plantarum were mainly of two kinds and this supports the hypothesis (Rogosa et al. 1953) that L. plantarum, as at present defined, might be a heterogeneous group consisting of more than one variety.

In contrast to the results obtained with the streptobacteria, the correlation between the vitamin requirements and the physiological characteristics of Lactobacillus brevis and $L$. buchneri strains was poor. A wide variety of vitamin-requirement patterns were obtained for these species, although no pattern was found to be common to both species. The suggestion of Rogosa et al. (1961) that the separation of $L$. brevis and $L$. buchneri, dependent physiologically on the fermentation of melezitose only, could be achieved by use of nutritional data was not supported because, contrary to their observations, many strains of $L$. brevis were found to have a requirement for riboflavin, and folic acid was necessary for the growth of most of the $L$. buchneri strains examined.

A requirement for thiamine by heterofermentative species of lactobacilli and the lack of this requirement by homofermentative species was again confirmed as a very reliable differentiating character, and the usefulness of nutritional studies as an adjunct to the more traditional methods of classifying the lactobacilli was again demonstrated. 


\section{Nitrate reduction}

Table 5 confirms the ability of certain strains of Lactobacillus plantarum to reduce nitrates. Two strains of $L$. casei also reduced nitrate but the proportion of positive strains was very much less than with $L$. plantarum species. None of the strains of the other species tested exhibited nitratase activity. It is evident, therefore, that under conditions of low carbohydrate content and a relatively high $\mathrm{pH}$ value, some strains of lactobacilli are able to reduce nitrate; failure to do so cannot be regarded, therefore, as a typical property of the genus Lactobacillus.

\section{REFERENCES}

Costilow, R. N. \& Humphreys, T. W. (1955). Nitrate reduction by certain strains of Lactobacillus plantarum. Science, 121, 168.

Frankin, J. G. \& Sharpe, M. E. (1963). The incidence of bacteria in cheese milk and Cheddar cheese and their association with flavour. J. Dairy Res. 30, 87.

Man, J. C. De, Rogosa, M. \& Sharpe, M. E. (1960). A medium for the cultivation of lactobacilli. J. appl. Bact. 23, 130.

Perry, K. D. \& Sharpe, M. E. (1960). Lactobacilli in raw milk and in Cheddar cheese. J. Dairy Res. 27, 267.

Rogosa, M. (1961). Experimental conditions for nitrate reduction by certain strains of the genus Lactobacillus. J. gen. Microbiol. 24, 401.

Rogosa, M., Frankern, J. G. \& Perry, K. D. (1961). Correlation of the vitamin requirements with cultural and biochemical characters of Lactobacillus spp. J. gen. Microbiol. 25, 473.

Rogosa, M. \& Sharpe, M. E. (1959). An approach to the classification of the Lactobacilli. J. appl. Bact. 22, 329.

Rogosa, M., TrTTSLer, R. P. \& GeIB, D. S. (1947). Correlation of vitamin requirements and cultural and biochemical characteristics of the genus Lactobacillus. J. Bact. 54, 13.

Rogosa, M., Wiseman, R. F., Mrtchell, J. A., Disraely, M. N. \& Beaman, A. J. (1953). Species differentiation of oral lactobacilli from man including descriptions of Lactobacillus salivarius nov. spec. and Lactobacillus cellobiosus nov.spec. J. Bact. 65, 681 .

Sharpe, M. E. (1962). Taxonomy of the lactobacilli. Dairy Sci. Abstr. 24, 109. 